\title{
Filosofía de la tecnología, desde la pertinencia social
}

\section{Philosophy of technology, from social relevance}

\author{
Bolívar Cabrera Berrezueta ${ }^{1}$ \\ ${ }^{1}$ Universidad Católica de Cuenca \\ *bolivarcabrera@ucacue.edu.ec
}

DOI: https://doi.org/10.26871/killkana_tecnica.v3i3.585

\begin{abstract}
Resumen
Las ciencias en la medida que definían su objeto de estudio se independizaban de la filosofía, que fungía ser la madre de las mismas. Por ello, aparece la historia, filosofía, teología, psicología, tecnología, investigación, entre otras, desde una perspectiva disciplinar. Este trabajo reflexiona sobre la Filosofía de la tecnología, desde la pertinencia social, que inicia con un análisis de las categorías de la filosofía tradicional: ontología, epistemología, teleología y axiología, luego se precisa hechos y acontecimientos relevantes que han sido determinantes para el establecimiento de etapas de la historia de las ciencias en general. Las etapas históricas de la filosofía, literatura y de otras ciencias, son la antigua, premoderna, moderna y posmoderna. Los mensajes emitidos dentro del proceso comunicacional desarrollado por el ser humano, constituye otro de los ejes para establecer etapas como la oralidad, escritural y electrónica, que a su vez ésta se particulariza en analógica y digital, con la finalidad de fortalecer la fundamentación filosófica de cada una de estas etapas. La investigación, pese a su recorrido cronológico, desde los tiempos más remotos hasta la actualidad, responde a un enfoque diacrónico y trasciende a lo transdisciplinario; más aún, cuando se precisa hechos o acontecimientos, que han surgido en una etapa, constituyen lo relevante de la siguiente, o lo contrario que, perteneciendo a esta etapa, fue antecedente de la inmediata anterior. Se concluye con un aporte a la teoría científica con la conceptuación de la tecnología con pertinencia social, en cuanto, ésta tiene como fundamento filosófico su aprovechamiento en beneficio de la sociedad y del individuo, así como, ser eje trascendente en contribuir a los requerimientos, necesidades y problemas del contexto. Filosofía de la tecnología de ser la esperanza para reducir las brechas generadas entre naciones e individuos y de la misma tecnología.
\end{abstract}

Palabras clave: Fundamentos filosóficos, tecnología, pertinencia social, etapas históricas, mensajes.

\begin{abstract}
Sciences as they would define their object of study became independent of philosophy, which served as their mother. Therefore, history, philosophy, theology, psychology, technology, research, among others, now appear from a disciplinary perspective. This work reflects on the Philosophy of technology, from social relevance, which begins with an analysis of the categories of traditional philosophy: ontology, epistemology, teleology and axiology, then it requires relevant facts and events that have been decisive for the establishment of stages in the history of science in general. The historical stages of philosophy, literature and other sciences are the ancient, pre-modern, modern and postmodern. The messages issued within the communication process developed by the human being, constitutes another of the axes to establish stages such as oral, scriptural and electronic, which are particularized in analog and digital, to strengthen the philosophical foundation of each of these stages. The research, despite its chronological journey, from ancient times to these days, responds to a diachronic approach and transcends the transdisciplinary; moreover, when facts or events are required, that appears at one stage, constitutes the relevant of the next, or the opposite, that belonging to this stage, was antecedent of the immediate previous. It concludes with a contribution to scientific theory with the conceptualization of technology with social relevance, since it is, philosophically based on its use for the benefit of society and the individual, as well as being a transcendent axis in contributing to the requirements, context needs and problems. Technology philosophy is the hope to reduce the gaps generated between nations and individuals and technology itself.
\end{abstract}

Keywords: Philosophical foundations, technology, social relevance, historical stages, messages.

\section{INTRODUCCIÓN}

Mientras la filosofía es la ciencia que estudia conceptos generales como del ser, el devenir, la mente y el conocimiento (Bunge, 2007); la tecnología es la rama del conocimiento que se ocupa de diseñar objetos, artefactos, máquinas, procesos industriales, así como de la normalización y el diseño de la acción humana; y, la filosofía de la tecnología tiene como objeto de estudio el análisis de la naturaleza, los problemas, las condiciones y el alcance del conocimiento tecnológico.

La filosofía de la tecnología, según Aguilar (2011)[1] es el saber globalizador, analítico y comprensivo de los proce- 
sos tecnológicos que facilita presupuestos antropológicos, epistemológicos y axiológicos, con miras a la producción de análisis críticos e interpretativos.

En el presente artículo se busca un acercamiento a la filosofía de la tecnología, desde un enfoque de la pertinencia social.

Para cumplir con este objetivo se realiza un análisis holístico, que inicia con el tratamiento de los cuatro problemas fundamentales de la filosofía tradicional, que son: Del ser absoluto u ontología, del conocimiento o gnoseología, de los fines o teleología y de los valores o axiología. En este enfoque se evidencia las etapas históricas de la filosofía o épocas con sus principales hechos o acontecimientos relevantes que dieron identidad a cada etapa. Estas etapas o épocas son enfocadas desde una mirada de la tecnología, teniendo como aspecto particular la pertinencia, en tanto que es lo adecuado para una situación determinada o necesidad específica.

En este contexto se coincide con el pensamiento que la pertinencia es la necesidad de un propósito a lo apropiado de construir una orientación fundamental y efectiva que sea capaz de participar en una producción de conocimiento donde podamos aprehender un objeto de estudio [2]).

Como investigador se pretende presentar un estudio, que permita entender que toda tecnología tiene su correspondiente fundamentación filosófica que da sentido de pertenencia de todo lo construido por el hombre para satisfacer sus necesidades y requerimientos, más aún cuando la filosofía de la tecnología proporciona categorías fundamentales para determinar el ¿por qué? y el ¿para qué de la tecnología?

\section{Metodología}

La investigación metodología empleada en este artículo de revisión es el analítico-sintético para la construcción del marco teórico relacionado con las etapas de la historia de la filosofía y la tecnología; así como el histórico-lógico para la sistematización de regularidades de diferentes pensadores que dan sustento científico a la indagación realizada. También la modelación en cuanto permite al investigador representar en forma de diagrama las preguntas con el impacto dinámico y social de cada etapa, así como las etapas, rasgos distintivos, consecuencias simultáneas y fundamento filosófico.

Para alcanzar el objetivo propuesto de ensayar una filosofía de la tecnología desde la pertinencia se alcanza con base en un enfoque diacrónico y transdisciplinario de la historia, filosofía y de la tecnología, incluso con algunas referencias de la literatura.

Ejes transversales en el artículo de revisión constituye las etapas de la historia de la filosofía, de los mensajes como generadores de grandes hechos relevantes para la historia, con la correspondiente fundamentación filosófica de la tecnología.

\section{Filosofía, Tecnología, Pertinencia}

La filosofía tradicional identificaba como problemas relevantes a su objeto de estudio en torno a problemas del ser, el conocimiento, fines y valores. Estos problemas corresponden a las categorías conocidas como ontología, epistemología, teleología y axiología, respectivamente.

\section{A. Problemas de la filosofía tradicional}

Resulta interesante ensayar o relacionar estas categorías, que fueran problemas fundamentales de la filosofía tradicional, al ámbito tecnológico.

Ontología que viene de las voces on, ontos, que significa ser y logos, equivalente a doctrina. Por tanto, la ontología es la rama de la filosofía que estudia la teoría sobre el ser, sobre su existencia, sus causas y sus efectos. En este caso específico se refiere al ser tecnología.

La tecnología, acorde a las tradiciones filosóficas, se ha ocupado de analizar la interacción del hombre con la técnica y la máquina en el contexto de la revolución técnicocientífica de la revolución industrial [3]. Posteriormente, en la primera mitad del siglo XX se orientó hacia una reflexión con una perspectiva crítica sobre los efectos que la técnica y sus avances tenían sobre la vida de los hombres en sociedad. En la actualidad la tecnología se debe entender como una construcción social, como un sistema que se compone no sólo del desarrollo de artefactos sino de elementos simbólicos, de tensiones, de valores sociales, de ideologías, de ambigüedades, de dualidades, como un sistema dinámico, multidireccional, interconectado y complejo. Finalmente, es necesario precisar que la principal acción de la tecnología está hacia el aprovechamiento de las tecnologías en la sociedad actual.

Epistemología procede de episteme que significa ciencia y logos igual a tratado. Es la historia o el estudio de los procesos cognitivos o de la sabiduría y del conocimiento. También se considera a la epistemología o gnoseología como el tratado o doctrina acerca del saber, los fundamentos y métodos del conocimiento.

En el caso de la epistemología de la tecnología corresponde al estudio de las relaciones de las categorías: conocimiento, ciencia, tecnología; las semejanzas y las diferencias de las categorías; y, la función o funciones de cada una de ellas.

Según el diccionario filosófico marxista (1984) el conocimiento es aquella categoría que toma por base la realidad objetiva del mundo exterior, que existe en forma independiente de la conciencia de los hombres, y considera su conocimiento como reflejo del mundo objetivo.

Con base en este principio, el mundo exterior es perfectamente cognoscible. La ciencia, en cambio, es la suma, el conjunto de los conocimientos sobre la naturaleza, la sociedad y el pensamiento, acumulados en el curso de la vida histórico-social. La tecnología, según el diccionario de Bunge (2007)[4], se basa en la ciencia, en tanto que busca nuevos conocimientos con potencial práctico y puede perfeccionarse con la ayuda de la investigación. 
Teleología se refiere a los fines. El término teleología deriva de telos que significa fin y logos, igual a doctrina; por tanto, se colige que es el tratado de los fines o de las causas finales.

La teleología es la concepción que las cosas, objetos, los seres tienen fines naturales, intrínsecos a cada uno ellos. La teleología tecnológica puede ser: inmanente o trascendente, en tanto, que posee fines concretos y privativos; $\mathrm{y}$, radical o moderada, en tanto que la tecnología está para contribuir a satisfacer las necesidades y requerimientos del ser humano.

Axiología es aquella parte de la filosofía que refiere a la doctrina de la teoría de los valores o las reflexiones, acerca del valor de la tecnología. Entre las variedades de las clases de valores, está la absolutista, la relativista, la objetiva, la subjetiva, la emotiva y la cognitiva.

La tecnología tiene un coste objetivo, en tanto que refiere en su aprovechamiento en todos los sectores sociales, morales, estéticos o para el desarrollo cognitivo. Es subjetiva o emotiva, en tanto que responden a la concepción del individuo o de la persona dependiendo de las situaciones o momentos históricos y de las relaciones de clase a de los grupos sociales. Al respecto, el pensamiento acerca que la axiología precautela los buenos hábitos del aprovechamiento de las dinámicas y tan veloces tecnologías, o como en la actualidad se denomina la realización de "buenas prácticas" [5].

Aspecto trascendente es que la tecnología, ya sea, desde las categorías de la ontología, epistemología, teleología y axiología sea pertinente. Es decir, que responda a las necesidades de las regiones, países, instituciones y al imperativo de responder a las expectativas de la sociedad en la que opera el individuo.

\section{B. Fundamentación filosófica de la tecnología}

El hombre ocupa el centro de las ciencias que se relacionan con la sociedad y el comportamiento humano: mientras la historia trata del desarrollo social y cultural, la filosofía pretende conocer la totalidad de la realidad o la concepción del mundo, la tecnología se ocupa de diseñar los artefactos y procesos de la acción del ser humano.

Para evidenciar los fundamentos filosóficos de la tecnología a lo largo de la historia, conlleva desarrollar un enfoque diacrónico, es decir un análisis que respete los ejes de sucesiones de los hechos o acontecimientos, a lo largo de la cronología del tiempo; y, de otra parte, con un enfoque que abarca varias disciplinas en forma transversal en cada etapa.

\section{Historia y tecnología}

La historia atestigua la existencia de importantes pueblos de la cultura oriental, entre los que sobresalen la India, China, Egipto, Babilonia e Israel con aportes significativos para el desarrollo de la humanidad. En occidente se da el florecimiento y desarrollo del pensamiento filosófico, literario, de las letras, el arte de los griegos y romanos, así como otros acontecimientos relevantes ocurridos en las etapas: Antigua, Media, Moderna y Posmoderna.

Edad Antigua: Según se desprende de los cuadros sincrónicos, que recoge sucesos históricos relevantes del Diccionario Sopena de Literatura Parnaso, la edad Antigua iniciaría en el año 4000 con los egipcios, que generaron los signos jeroglíficos, como evidencia de la generación de diferentes formas gráficas de comunicación del ser humano, que constituyen los primeros registros de la historia del hombre en la faz de la tierra, hasta el año 476 que corresponde al fin del Imperio Romano de occidente [6]. Etapa que la denominación clásica por los grandes aportes a la ciencia moderna, el conocimiento, las artes y la tecnología.

Etapa antigua que se caracteriza por el aparecimiento de la filosofía, como la madre de las ciencias. En Grecia, concretamente en la región de Mileto, con Tales, Anaximandro, Anaxímenes surgen los primeros sistemas de conocimiento, como explicación racional del universo, como teoría acerca de la naturaleza por el principio natural y sin que explicación repercuta en lo religioso, la técnica o la política [7]. Los primeros filósofos griegos ya distinguían entre el simple hacer por la experiencia del saber - hacer con conocimiento de causa o técnica y del hábito del saber [8]. Los griegos diferenciaban entre el saber hacer por la experiencia y el saber hacer con arte, técnica, táctica y pericia.

La técnica, en esta etapa, tiene su sustento filosófico la búsqueda de la verdad, del saber o por conocer; que los objetos, hechos, acontecimientos y los nuevos conocimientos se dan en un contexto determinado y están para responder a las necesidades inmediatas y trascendentales para su existencia y supervivencia. Es decir, saber hacer con juicio, discernimiento y conocimiento de causa.

Luego aparecen los aportes de: Sócrates con el descubrimiento de los razonamientos inductivos y la definición universal; Platón con la dialéctica para alcanzar el conocimiento y sus concepciones, sobre el verdadero conocimiento, habrá de versar sobre la verdadera realidad (gnoseología) y la preocupación por el verdadero ser, la realidad auténtica (ontología); y, Aristóteles con las tres formas de conocimiento: la experiencia, ciencia e inteligencia. Al referirse a la experiencia expresa este filósofo que se halla el arte o técnica y que el conocimiento técnico sabe ya lo que es la cosa, pero ignora todavía el porqué de la misma.

Edad Intermedia entre la antigua y los tiempos modernos. En occidente es la edad comprendida entre el 476, que corresponde al fin del Imperio Romano de Occidente, hasta al 1453 con la caída de Constantinopla en poder de los turcos o fin del imperio Bizancio.

Los aportes de esta etapa son proporcionados por la escolástica, instituciones eclesiásticas, especialmente conventuales, catedralicias, de las que surgieron las primeras universidades, con la enseñanza de las artes, las materias literarias y las científicas.

Los progresos intelectuales, técnicos y económicos [9] van a expandirse por el mundo entero con el descubrimiento 
de América: progreso de la agricultura, urbanización, del estado, del derecho. Es la edad gestora y constructora del viejo continente en su afán conquistador, de la producción industrial y de la comercialización generalizada.

El pensamiento la Edad Media "se presenta entonces como la matriz del progreso histórico, un período de acumulación progresiva de un capital que será en seguida mundializado" en la etapa de los tiempos modernos.

Edad de los tiempos o acontecimientos modernos o edad Moderna. Desde la caída de Constantinopla (1453) inicia la etapa de los tiempos modernos, caracterizada por diferentes descubrimientos geográficos, la aparición de la máquina de vapor, de los ferrocarriles, la invención de la imprenta, la revolución industrial. En el orden cultural y filosófico esta edad se abre con el Renacimiento, como la gran revolución de la ciencia. Están los aportes de Leonardo de Vinci, Nicolás Copérnico, Galileo Galilei, Nicolás Maquiavelo, entre otros.

El pensamiento multifacético de Leonardo da Vinci, los descubrimientos geográficos, los descubrimientos astronómicos de Galileo Galilei, la teoría heliocentrista de Nicolás Copérnico, las nuevas concepciones de ciencia política de Nicolás Maquiavelo, así como el desarrollo de las fuerzas productivas, la técnica y de los medios de producción constituyen los nuevos hitos del proceso tecnológico.

A partir de la segunda mitad del siglo XVIII se inicia una profunda transformación de la estructura económica conocida como revolución industrial (Carrillo, 2017) está contemplada dentro de tres revoluciones de la modernidad: la primera con la máquina de vapor, la producción textil y el acero; la segunda, con el uso de la ciencia y fomento de la innovación; y, la tercera, la científica y tecnológica. Etapa que se consolida el racionalismo, el empirismo, la ilustración, romanticismo, racionalismo crítico e idealismo. Se cierra este auge inventivo en el año 1789 con la toma de la Bastilla.

Edad de los acontecimientos posteriores a la modernidad o Posmoderna. Desde la promulgación de los principios de la revolución francesa de libertad, igualdad, confraternidad, solidaridad (1789), hasta los días actuales corresponde la edad posmoderna. Algunos historiadores denominan edad contemporánea, otros, como Giddens, Bauman, Luhmann y Beck (1996) se refiere como modernidad superior o tardía, tiempo social tardomoderno. Castells (2006) caracteriza esta etapa por la gran producción de información e innovación y la denomina como la "era de la información" (p. 58).

En los últimos tiempos, el fin último de las tecnologías se orienta al logro de la eficacia por la eficacia (Aguilar, 2011). En otros términos, este fin, es la capacidad de generar conocimiento, aprovechamiento de las tecnologías de información y comunicación, internet, simulaciones, digitalización, entornos virtuales con los recursos y tiempos necesarios.

\section{Los mensajes y la tecnología}

Los mensajes emitidos en el proceso comunicacional constituyen otra de las formas para establecer etapas o edades con base a considerar los hechos y acontecimientos relevantes generados por la especie humana, acordes al desarrollo tecnológico.

McLuhan (1995) fundamenta su pensamiento sobre los mensajes y la manera de revelar, caracterizar y valorar los cambios culturales de la sociedad con base en el tétrade, que es un diagrama mental, que identifica cuatro momentos para evidenciar el impacto dinámico y social de una determinada época: a) Intensificación de hechos relevantes; b) Precisión de lo que se vuelve obsoleto; c) Recuperación de algún factor dejado de lado desde tiempo atrás; y, d) Modificaciones en dirección de un efecto opuesto. Véase la figura 1

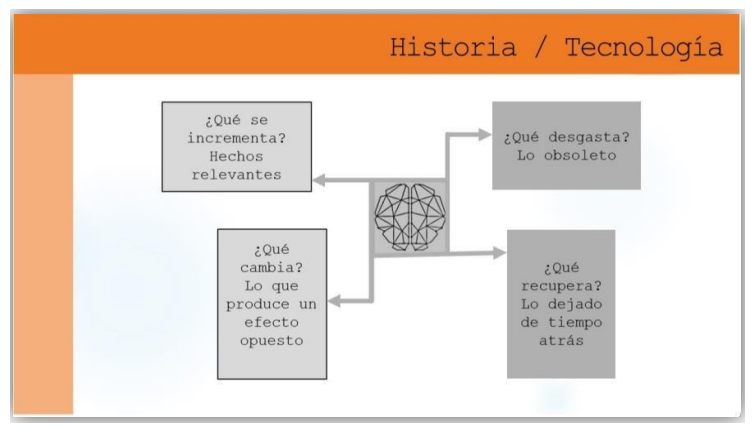

Fig. 1: Tétrade; Preguntas y acciones

Desde los sistemas de comunicación del ser humano, existen hechos y acontecimientos trascendentales que determinan la existencia de etapas con sus particularidades comunicativas. McLuhan y Bruce R. Powers establecen tres etapas a las que denominan: de la oralidad, de la escritura y electrónica.

Etapa de la oralidad o primer modo de comunicación complejo: El hombre primitivo, por su esencia humana, tuvo como primera necesidad la comunicación con sus semejantes y con el entorno en el que se circunscribe su accionar humano.

La etapa de la oralidad corresponde a la sociedad primitiva en la que se practica la transmisión de mensajes en forma oral, de cara a cara, luego se da la representación de mensajes en pictogramas, jeroglíficos y cuneiforme como el "Código de Hamurabi", escrita hacia el año 1700 antes de Cristo.

El Libro de los Vedas con sus partes: Rig-Veda, YájurVeda, Sama-Veda, Atharva-Veda, escrito entre el 2100 y 1400, antes de Cristo, constituye la expresión del lenguaje articulado y es la obra literaria más antigua del mundo, que se transmitía en forma oral y de generación en generación, como una tradición del pueblo Hindú.

Grandes dramaturgos de la historia del mundo occidental, en la época presocrática y el mismo Sócrates (469399) se constituye en paradigmas o modelos de la oralidad. 
Sin embargo, la creación del alfabeto fonético será causa determinante para el desuso de la cultura oral.

Esta etapa de la palabra oral es vista como un don otorgado por el creador para ayudar al hombre en su quehacer en este mundo terrenal y Avanza hasta entrada la edad que media entre la antigua y la moderna.

Hacia 1995 McLuhan en su diálogo con Powers, expresa "El mundo oral responde a lo simultáneo, lo holístico, lo armonioso (es literalmente la morada de la canción, para nosotros" (p. 137), como expresión de la importancia del mensaje a través de la palabra oral.

Etapa escritural: La necesidad humana de registrar el mensaje articulado y estético, el discurso, así como compartir información, contribuye al origen y a vigorizar la escritura.

La escritura era una técnica que con base en el papel generaba control e informaciones voluminosas a largas distancias con comunicaciones militares y diplomáticas. Entre el 300 y 200 antes de Cristo aparecen las epopeyas: "El Mahabharata" y el "El Ramayana" escritos en idioma sánscrito.

La prensa manual de la imprenta, inventada por Johannes Gutenberg (1399-1468), primero con los tipos de madera, luego metálicos, evidencia el aparecimiento del libro impreso. El apogeo de las letras coincide con la aparición de la obra "El ingenioso hidalgo don quijote de la mancha" (1605) de Miguel de Cervantes Saavedra, principal representante de la edad de oro de la literatura española. Gutenberg da inicio a la etapa de la imprenta y con ello el desarrollo de la cultura europea y luego mundial.

La imprenta y el correo postal marcaron un antes y un después en esta etapa, signada por la industrialización de la economía, la migración urbana y la formación de la sociedad de masas.

Etapa de comunicación electrónica: Con base en las tecnologías ultra veloces (ibídem, p. 13) se pueden utilizar para postular posibles futuros, como un artefacto con uso totalmente saturado podría producir lo inverso de la intención original, hasta llegar a generar un nuevo invento que transforme la sociedad. Esa fue la realidad, en su respectivo momento con la radio, el filme, las grabaciones, el teléfono, el cine, la telegrafía, el facsímil, la televisión, la internet, las redes sociales, las simulaciones, entre otras tecnologías de información y comunicación que abren grandes expectativas del desarrollo tecnológico.

Esta etapa de comunicación electrónica, con la finalidad de una eficiente sistematización, se la divide en sistemas de comunicación analógica y sistema digital.

Los sistemas de comunicación analógica con grandes inventos, fruto del resultado de una serie de descubrimientos e ideas, entre otros: El telégrafo para la transmisión de mensajes, primero con Orsted (1820) y luego Morse, con el sistema de telegrafía eléctrica (1844); el teléfono por Bell (1876) para transmitir sonidos a distancia; la radio con la primera fundamentación teórica por Maxwell (1875), luego Marconi (1896) envía señales desde una oficina a otra; y, la transmisión de imágenes a distancia por Baird (1922). Inventos que ofrecen nuevas formas de comunicación a nivel de toda la sociedad, que a su vez se constituyen en antecedentes para otros inventos.

Corresponde a los inicios de la revolución industrial, que a su vez será el tránsito a hacia la energía eléctrica, electrónica y atómica. Aparecen las primeras PCs, que constituyen los antecedentes de la sociedad de la información y comunicación.

El sistema de comunicación digital o del escenario de la internet, con otras denominaciones como la revolución de las pantallas, de las plataformas virtuales y la comunicación a través de las redes sociales y sistemas complejos integrados para las diferentes actividades que realiza el ser humano.

Corresponde esta etapa a la actual revolución científica e intelectiva. Es la era del conocimiento, de la industria basada en el poder de la mente, de la tecnología de última generación. La etapa digital corresponde a la sociedad del conocimiento, de la innovación, de la tecnología, en busca de la integración para el aprovechamiento eficiente. Las fundamentaciones presentadas se sistematizan en la tabla I

TABLA I: Etapas, rasgos distintivos, consecuencias simultáneas y fundamento filosófico

\begin{tabular}{|c|c|c|c|}
\hline ETAPA & $\begin{array}{c}\text { RASGOS } \\
\text { DISTINTIVOS } \\
\text { (Tétrade) }\end{array}$ & $\begin{array}{l}\text { CONSECUENCIAS } \\
\text { SIMULTÁNEAS }\end{array}$ & $\begin{array}{c}\text { FUNDAMENTO } \\
\text { FILOSÓFICO }\end{array}$ \\
\hline \multirow{4}{*}{ Oralidad } & a) Intensificará algo & $\begin{array}{l}\text { - El alfabetismo en el mundo } \\
\text { - Son oidores (que escuchan) } \\
\text { - Responden a lo simultáneo, lo } \\
\text { holístico, lo armonioso }\end{array}$ & \multirow{4}{*}{$\begin{array}{l}\text { - Dogmatismo, mítica- } \\
\text { religiosa } \\
\text { incondicional } \\
\text { - Clasicismo } \\
\text { tradición ordad se da por la } \\
\text { misticismo, la intuición }\end{array}$} \\
\hline & $\begin{array}{l}\text { b) Hará que algo se torne } \\
\text { obsolete }\end{array}$ & $\begin{array}{l}\text { Tradición generacional (fidelidad oral) } \\
\text { - Burocracia sacerdotal }\end{array}$ & \\
\hline & c) Recuperará algo & $\begin{array}{l}\text { - Manuscritos leídos (voz alta) } \\
\text { - Fortalecimiento de la memoria } \\
\text { - Principio de causalidad } \\
\text { - Oralidad como forma de arte: } \\
\text { - El poder de la palabra }\end{array}$ & \\
\hline & $\begin{array}{l}\text { d) Empujará a un efecto } \\
\text { opuesto }\end{array}$ & - Alfabetismo escrito & \\
\hline \multirow[t]{4}{*}{ Escritural } & a) Intensificará algo & $\begin{array}{l}\text { - Sistematización del lenguaje } \\
\text { - Normas para la pronunciación y el } \\
\text { significado }\end{array}$ & \multirow{4}{*}{$\begin{array}{l}\text { - Mentalidad centrada } \\
\text { en la naturaleza } \\
\text { humana. } \\
\text { - Antropocentrismo }\end{array}$} \\
\hline & $\begin{array}{l}\text { b) Hará que algo se torne } \\
\text { obsolete }\end{array}$ & $\begin{array}{l}\text { - Los copistas o escribas } \\
\text { - Burocracias de los templos } \\
\text { - Élites del conocimiento (burocracia } \\
\text { militar) }\end{array}$ & \\
\hline & c) Recuperará algo & $\begin{array}{l}\text { - Expansionismo y conquistas } \\
\text { - Producción cuantitativa y difusión de } \\
\text { la información } \\
\text { - Evidencia del texto escrito }\end{array}$ & \\
\hline & $\begin{array}{l}\text { d) Empujará a un efecto } \\
\text { opuesto }\end{array}$ & $\begin{array}{l}\text { - Tecnología analógica (radio, filme, la } \\
\text { grabación. }\end{array}$ & \\
\hline \multirow{4}{*}{ Virtual } & a) Intensificará algo & - Los modos visual y acústico & \multirow{4}{*}{$\begin{array}{l}\text { Acercarse al presente } \\
\text { como tarea, como } \\
\text { medio a ser analizado, } \\
\text { discutido, tratado para } \\
\text { un futuro con mayor } \\
\text { claridad }\end{array}$} \\
\hline & $\begin{array}{l}\text { b) Hará que algo se torne } \\
\text { obsolete }\end{array}$ & $\begin{array}{l}\text { - El habla, la escritura } \\
\text { - Obsoleta: la misma tecnología }\end{array}$ & \\
\hline & c) Recuperará algo & $\begin{array}{l}\text { - Los mensajes se expanden al contexto } \\
\text { social (brechas) }\end{array}$ & \\
\hline & $\begin{array}{l}\text { d) Empujará a un efecto } \\
\text { opuesto }\end{array}$ & $\begin{array}{l}\text { - Las áreas audio, visual y táctil } \\
\text { - Tecnología digital }\end{array}$ & \\
\hline
\end{tabular}

Los acontecimientos y hechos históricos desde un enfoque diacrónico y ransdisciplinario, así como desde los mensajes deben ser analizados desde un enfoque de pertinencia social. 


\section{TECNOLOGÍA CON PERTINENCIA SOCIAL}

A partir del pensamiento que la tecnología "no sólo constituye un conjunto de herramientas, sino un entorno - un espacio, un ciberespacio - en el cual se producen las interacciones humanas" [10] y que la tecnología en muchos de los casos induce a gastar dinero en la adquisición de nuevos recursos técnicos ocultando el hecho de que se crean con ello más problemas de los que se resuelven, de que las posibilidades de las nuevas tecnologías aumentan la necesidad de actuar con imaginación, planificar con cuidado y superar sobre la marcha desafíos impensados.

La tecnología debe estar lejos de tecnofilia y de la tecnofobia, así como también de la infoxicación y la infobesidad como un problema de fondo por los malos hábitos de consumo digital-informativo y de interrelaciones sociales [11]. Argumentos que fortalece la presente investigación acerca de la filosofía de la tecnología desde un enfoque de la pertinencia.

Afín al pensamiento sobre la promoción de una mayor vinculación de la universidad con el Estado y con todos los sectores de la sociedad y la incorporación de las llamadas nuevas cultura como de la calidad, la evaluación, la tecnología se debe incorporar la cultura de la pertinencia social [12] y del pensamiento de [13] que la pertinencia como adaptabilidad a las necesidades de las sociedades, en contextos culturales y sociales.

Con los antecedentes expuestos la tecnología es pertinente en cuanto responde a las expectativas y necesidades de la sociedad, a las tendencias demográficas, así como a los requerimientos del contexto regional, nacional, local, al desarrollo científico, humanístico, a la diversidad cultural, a los sectores históricamente excluidos. Mayor será su pertinencia en la medida que la tecnología contribuya en la reducción no sólo las brechas digitales, sino sobretodo reduzca las brechas económicas, entre países e individuos, que contribuya en la solución de los problemas demográficos, así como en la conservación de la naturaleza, de los bosques, que constituyen los pulmones del mundo. De igual manera, la pertinencia tecnológica debe precautelar la equidad y los principios éticos para un verdadero aprovechamiento de la tecnología en beneficio de la sociedad y de todos los individuos sin importar el género, la condición económica, social, política, cultural, nivel educativo, ni creencias, como derecho pleno en su existencia.

\section{CONCLUSIONES}

El objetivo propuesto de realizar una fundamentación filosófica de la tecnología se ha conseguido con base a determinar hechos y acontecimientos relevantes desarrollados por el ser humano con un enfoque diacrónico y transdisciplinario de ciencias como la historia, la filosofía, entre otras áreas del conocimiento.

La etapa antigua se caracteriza por el aparecimiento de la filosofía, que se constituye la madre de otras ciencias. Mientras que la etapa de la modernidad, como generadora de revoluciones como la industrial, la analógica y la digital.
Esta etapa está presidida por un largo período, de caso un siglo (476 - 1453), en la que se desarrolla la teología, la enseñanza escolarizada, la creación de universidades. El gran desarrollo de la tecnología, así como de los sistemas comunicacionales da origen a la denominada era de la información y de la comunicación.

Desde los mensajes en el proceso comunicacional se ha asumido el pensamiento de McLuhan sobre tres etapas de la historia: la oral, la escritura y la virtual, para relievar la importancia de hechos y acontecimientos desde los mensajes.

La filosofía de la tecnología tiene como sustento filosófico el aprovechamiento de las tecnologías en el campo educativo, de ser pertinente para satisfacer los requerimientos de la sociedad, de la conservación del medio ambiente, para reducirlas brechas económicas, demográficas, de personas con discapacidades y en general de las diferentes actividades que realiza el ser humano.

\section{REFERENCIAS}

[1] F. Aguilar-Gordón, "Reflexiones filosóficas sobre la tecnología y sus nuevos escenarios," Sophía, no. 11, pp. 123-172, 2011.

[2] . Espinosa, M. E. Luna, and V. Mata, "Pertinencia y uso del marco teórico en la investigación," in Maestría en tecnología educativa. Módulo propedéutico, (México, D.F), pp. 443-465, México: ILC, 1992.

[3] J. Tabares-Quiroz and S. Correa-Vélez, "Tecnología y sociedad: una aproximación a los estudios sociales de la tecnología," CTS: Revista iberoamericana de ciencia, tecnología y sociedad, vol. 9, no. 26, pp. 129144, 2014.

[4] M. Bunge, Diccionario de filosofía. México, D.F.: México: Siglo XXI, cuarta ed., 2007.

[5] C. Cobo-Romaní and J. W. Moravec, Aprendizaje invisible: Hacia una nueva ecología de la educación, vol. 3. Edicions Universitat Barcelona, 2011.

[6] M. Armiño, Parnaso: diccionario Sopena de literatura, vol. 2. R. Sopena, 1988.

[7] A. González-Alvarez, Manual de historia de la filosofía. Gredos, 1971.

[8] L. Sarramona, "Ciencia y tecnología en educación," in Maestría en tecnología educativa. Módulo propedéutico (. M. d. L. M. Martínez, F. Fierro, ed.), pp. 25-36, México, D.F.: México: ILCE, 1992.

[9] O. Boulnois, “¿ qué hay de nuevo? la edad media," Universitas Philosophica, vol. 33, no. 67, pp. 321350, 2016.

[10] N. C. Burbules, Educación: riesgos y promesas de las nuevas tecnologías de la información. Ediciones Granica SA, 2006.

[11] L. M. R. Rodríguez, I. Aguaded, and W. F. Gadea, "Crear conciencia informativa: usos y abusos del consumo de información en la era digital," in Desafios en educación superior, pp. 153-165, Universidad Nacional de La Matanza;, 2016. 
[12] J. Gacel-Ávila, "Educación superior, internacionalización e integración en américa latina y el caribe," in P. Henríquez, Tendencias de la educación superior en América Latina y el Caribe 2018. Conferencia Regional de Educación Superior-CRES, pp. 111-169, 2017.

[13] L. Cotino-Hueso, El derecho a la educación como derecho fundamental. Especial atención a su dimensión social prestacional. Centro de Estudios Políticos y Constitucionales, 2012.

Recibido: 25 de septiembre de 2019

Aceptado: 12 de noviembre de 2019 
